\title{
Bragg scattering of surface plasmon polaritons on extraordinary transmission through silver periodic perforated hole arrays
}

\author{
Ming-Wei Tsai, Tzu-Hung Chuang, Hsu-Yu Chang, and Si-Chen Lee ${ }^{\text {a) }}$ \\ Department of Electrical Engineering, Graduate Institute of Electronics Engineering, \\ National Taiwan University, Taipei, Taiwan 106, Republic of China
}

(Received 13 December 2005; accepted 26 April 2006; published online 24 May 2006)

\begin{abstract}
Extraordinary optical transmission through a two-dimensional periodic perforated Ag film in the far infrared region was demonstrated. When the squared hole size is close to a half lattice constant $a / 2$, the split of the degenerate $( \pm 1,0) \mathrm{Ag} / \mathrm{Si}$ and $(0, \pm 1) \mathrm{Ag} / \mathrm{Si}$ modes into two peaks becomes apparent. Surface plasmon polaritons dispersion relations with variously sized square holes are measured to investigate the different surface charge fields at the periodic metal array. Strong scattering of the forward SPP waves, in the $(1,0) \mathrm{Ag} / \mathrm{Si}$ mode, leads to a much lower transmission than that of in the $(-1,0) \mathrm{Ag} / \mathrm{Si}$ mode. Experimental results demonstrate that the photonic band gap opens up when the size of the squared hole exceeds a half lattice constant $a / 2$. (C) 2006 American Institute of Physics. [DOI: 10.1063/1.2206553]
\end{abstract}

Metal films with two-dimensional subwavelength periodic perforated hole arrays exhibit extraordinary optical transmission. ${ }^{1,2}$ When a single slit in a metallic film is surrounded by periodic surface corrugations, the emitted light field from the slit concentrates in a specific direction with a constrained range of angles, instead of diverging in all directions, which supports the directional beaming nature of the surface plasmon. ${ }^{3,4}$ If the period of the corrugation is appropriate, then the surface plasmon plaritons (SPPs) can Bragg reflect and an energy gap opens up in the SPP dispersion relation. ${ }^{5,6}$ Recent work on the conservation of surface plasmons and light through periodic perforated hole arrays ${ }^{7}$ has elucidated the propagation of surface plasmons. Extraordinary optical transmission in the middle and far infrared region has been demonstrated. ${ }^{8-10}$ They have potential applications in optical filters and optical modulators. This work investigates how hole size affects extraordinary transmission through a two-dimensional periodically perforated $\mathrm{Ag}$ film in the far infrared region. SPP dispersion relations with variously sized square holes are measured to discuss the various surface charge displacements on periodic perforated Ag film.

After the photoresist was spun onto a silicon wafer and following pattern transfer, $50 \mathrm{~nm}$ thick $\mathrm{Ag}$ metal films with square holes of various sizes were deposited and lifted off. A Bruker IFS $66 \mathrm{v} / \mathrm{s}$ system was adopted to measure zeroorder transmission spectra. The sample is defined to lie in the $(x, y)$ plane, and is rotated about the $y$ axis in $1^{\circ}$ increments up to $\theta=50^{\circ}$. The light is incident in the $z$ direction, allowing the dispersion relation in the $\mathbf{k}_{x}$ direction to be studied. The wave number resolution of the measurement was $8 \mathrm{~cm}^{-1}$.

The conservation of momentum for surface plasmons ${ }^{1}$ is given by

$$
\mathbf{k}_{\mathrm{sp}}=\mathbf{k}_{x}+i \mathbf{G}_{x}+j \mathbf{G}_{y},
$$

where $\mathbf{k}_{\mathrm{sp}}$ is the surface plasmon wave vector given by

\footnotetext{
${ }^{a)}$ Electronic mail: sclee@cc.ee.ntu.edu.tw
}

$$
\left|\mathbf{k}_{\mathrm{sp}}\right|=\frac{\omega}{c}\left(\frac{\varepsilon_{1} \varepsilon_{2}}{\varepsilon_{1}+\varepsilon_{2}}\right)^{1 / 2},
$$

where $\omega$ is the frequency of surface plasmon that is excited by the incident radiation with frequency $\omega ; \mathbf{k}_{x}=\left|\mathbf{k}_{0}\right| \sin \theta$; $\left|\mathbf{k}_{0}\right|=2 \pi / \lambda$ is the wave vector of the incident radiation, and $\lambda$ is the wavelength in vacuum. $\varepsilon_{1}$ is the dielectric constant of the interface medium, and $\varepsilon_{2}$ is that of the metal. $\mathbf{G}_{x}$ and $\mathbf{G}_{y}$ are the reciprocal lattice vectors of a square lattice with $\left|\mathbf{G}_{x}\right|=\left|\mathbf{G}_{y}\right|=2 \pi / a$, and $i$ and $j$ are integers. For normal incident light, $\mathbf{k}_{x}=0$, Eq. (2) reduces to

$$
\left(i^{2}+j^{2}\right)^{1 / 2} \lambda=a\left(\frac{\varepsilon_{1} \varepsilon_{2}}{\varepsilon_{1}+\varepsilon_{2}}\right)^{1 / 2},
$$

The real part of the dielectric constants of $\mathrm{Ag}$ at 18, 22, 24, and $30 \mu \mathrm{m}$ are $-1.59 \times 10^{4},-2.31 \times 10^{4},-2.71 \times 10^{4}$, and $-3.98 \times 10^{4},{ }^{11}$ respectively. That of $\mathrm{Si}$ is 11.7 at all of these wavelengths. Figures 1 and 2 show the zero-order transmission spectra at normal incidence with variously sized holes. The lattice constant $a$ is $7 \mu \mathrm{m}$; the square length $L$ varies from 3.8 to $4.5 \mu \mathrm{m}$ in Fig. 1. In Fig. 2, the square length $L$

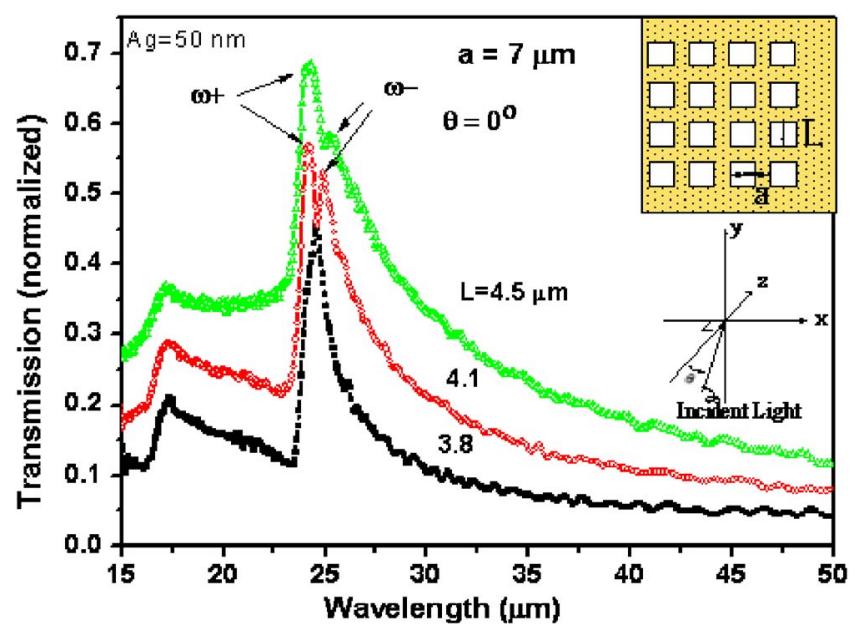

FIG. 1. (Color online) Zero-order transmission spectra at normal incidence with different squared hole size $L$. The Ag film thickness is $50 \mathrm{~nm}$ and the lattice constant $a$ is $7 \mu \mathrm{m}$. 


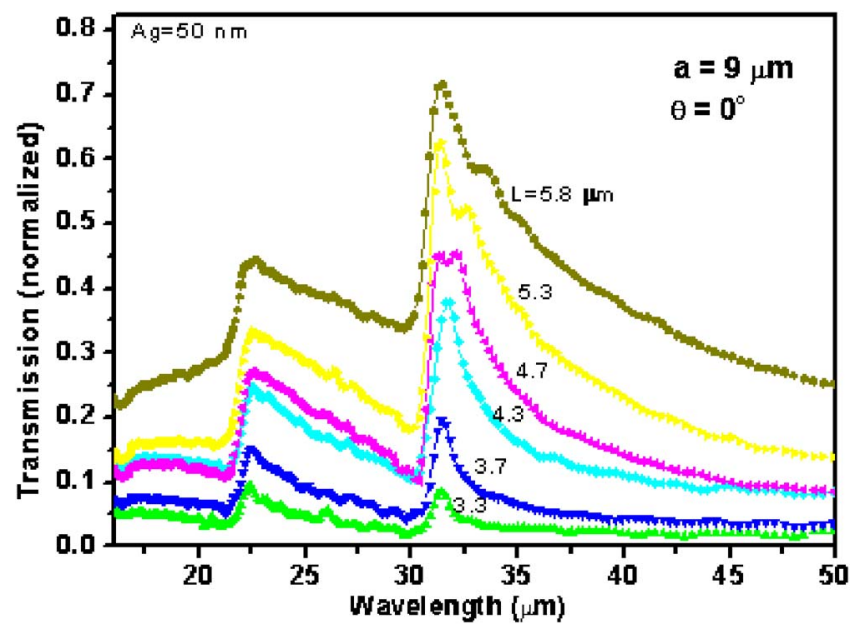

FIG. 2. (Color online) Zero-order transmission spectra at normal incidence with different squared hole size $L$. The Ag film thickness is $50 \mathrm{~nm}$ and the lattice constant $a$ is $9 \mu \mathrm{m}$.

varies from 3.3 to $5.8 \mu \mathrm{m}$, and $a$ is $9 \mu \mathrm{m}$. Figure 1 clearly shows that when the square hole is larger than half of the lattice constant $a / 2, L \geqslant 4.1 \mu \mathrm{m}$, the degenerate $( \pm 1,0)$ $\mathrm{Ag} / \mathrm{Si}$ and $(0, \pm 1) \mathrm{Ag} / \mathrm{Si}$ modes split into two peaks at 24 and $25 \mu \mathrm{m}$, respectively. At $L=3.8 \mu \mathrm{m}$, the split in the degenerate modes can barely be seen. The structure with a different lattice constant $a=9 \mu \mathrm{m}$ yields similar results, as shown in Fig. 2. The split double peaks represent degenerate modes that are composed of $(0, \pm 1) \mathrm{Ag} / \mathrm{Si}$ and $( \pm 1,0)$ $\mathrm{Ag} / \mathrm{Si}$ modes, because when the normal incident light propagates through the sample, Bragg scattering of SPPs on the periodic perforated square hole arrays yields two standing waves $\left(\omega_{+}\right.$and $\left.\omega_{-}\right)$- each with its own surface charge energy $y^{5,6}$ - and splits into two peaks. The photonic band gap opens up when the size of the squared hole exceeds a half lattice constant $a / 2$. The transmission intensity of $\omega_{+}$becomes larger than $\omega_{-}$as the size of holes increases, because as the size of the holes increases, such that the metal linewidth decreases, the $\omega_{+}$mode more easily increases the surface charge density with a single polarity in the metal line to a value that exceeds that required to form a charge dipole in the $\omega_{-}$mode. Figures 3(a)-3(d) display the SPP dispersion relation of the periodic arrays of square holes with lattice constant $a=9 \mu \mathrm{m}$ and squared hole sizes $L=6,5.8,4.7$, and $4.3 \mu \mathrm{m}$, respectively. At normal incidence $\left(\theta=0^{\circ}\right)$, fourfold degeneracy is evident at 0.04 and $0.058 \mathrm{eV}$, which comes from $\mathrm{Ag} / \mathrm{Si}( \pm 1,0)$ plus $\mathrm{Ag} / \mathrm{Si}(0, \pm 1)$ and $\mathrm{Ag} / \mathrm{Si}( \pm 1, \pm 1)$, respectively. In the $(1,0) \mathrm{Ag} / \mathrm{Si}$ mode, the transmission contrast is weak and the bright line that represents the transmission peak moves to higher energy as $\mathbf{k}_{x}$ increases. Figures 4(a) and 4(b) present the detailed transmission spectra at different incident angles of light. The lattice constant $a$ is $9 \mu \mathrm{m}$,

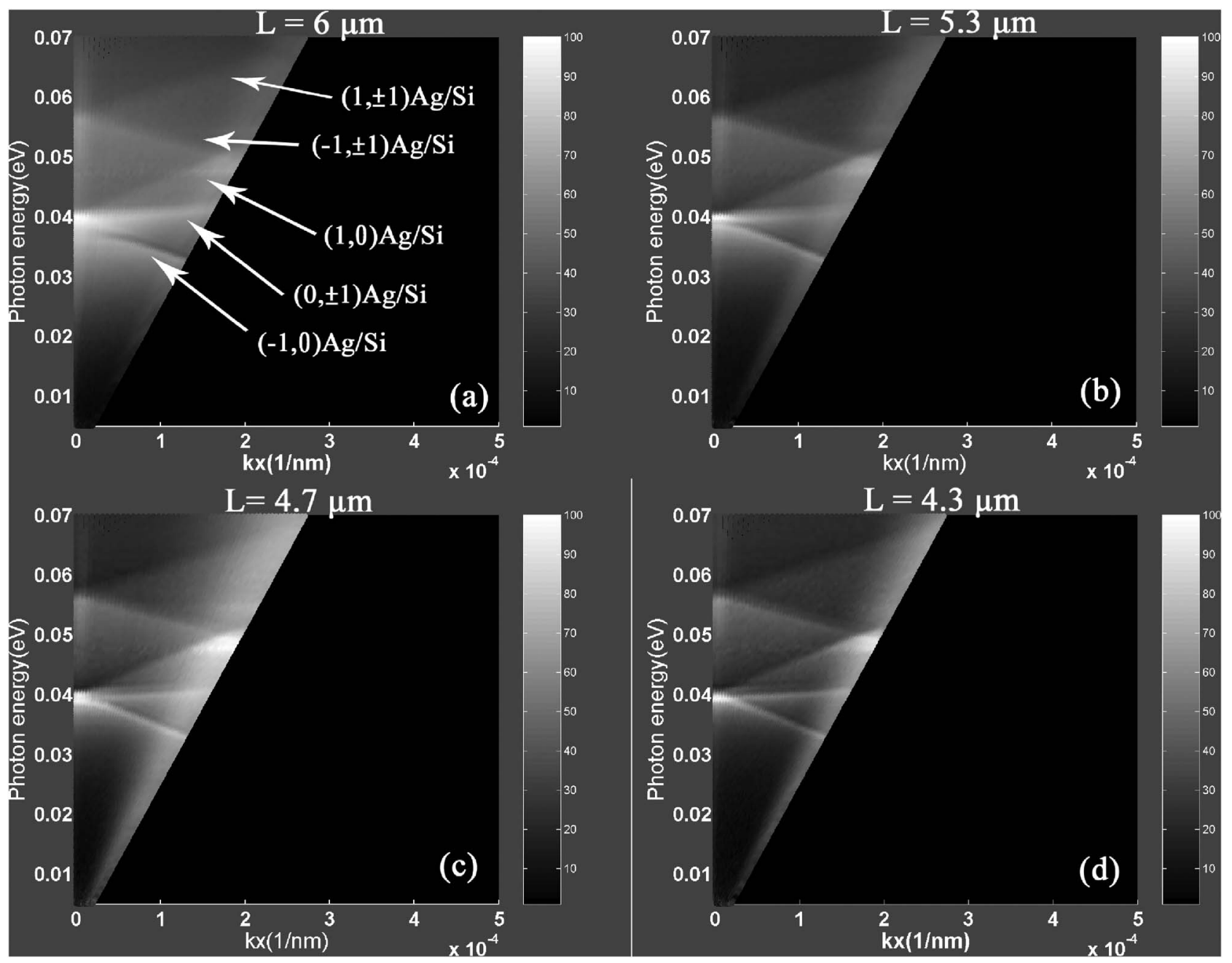

FIG. 3. Energy dispersion relation of SPPs as a function of $\mathbf{k}_{x}$, the transmission intensity is depicted with gray scale. The square hole length are (a) $6 \mu$ m, (b) $5.3 \mu \mathrm{m}$, (c) $4.7 \mu \mathrm{m}$, and (d) $4.3 \mu \mathrm{m}$, and the lattice constant of the hole array $a$ is $9 \mu \mathrm{m}$. 

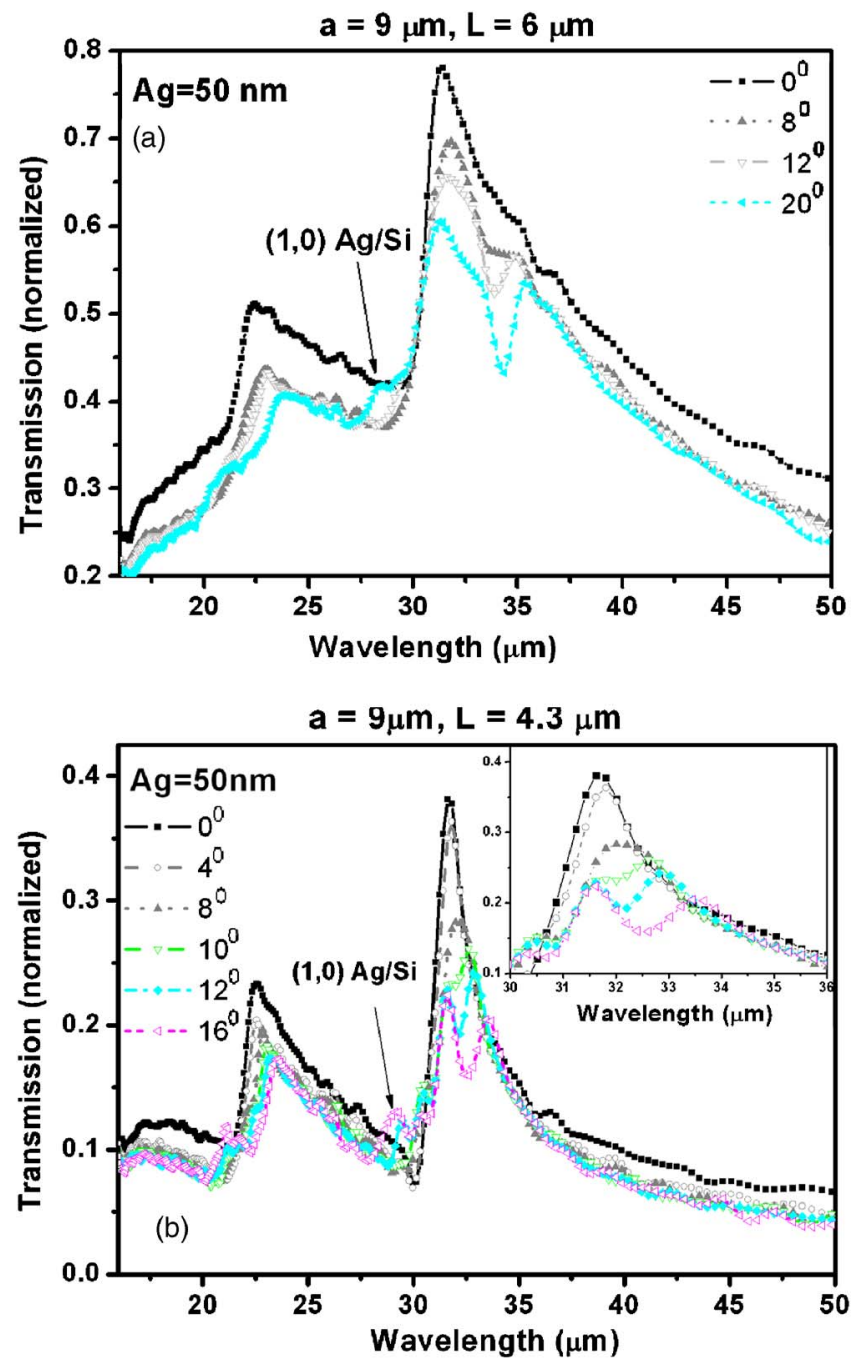

FIG. 4. (Color online) Transmission spectra of a periodic squared hole array with lattice constant $a$ of $9 \mu \mathrm{m}$ and square length $L$ of (a) $6 \mu \mathrm{m}$ and (b) $4.3 \mu \mathrm{m}$ at various incident angles.

and the square length $L$ varies from 4.3 to $6 \mu \mathrm{m}$. When the light incident angle or $\mathbf{k}_{x}$ increases, as shown in Figs. 4(a) and $4(\mathrm{~b}),(0, \pm 1) \mathrm{Ag} / \mathrm{Si},(-1,0) \mathrm{Ag} / \mathrm{Si}$, and $(1,0) \mathrm{Ag} / \mathrm{Si}$ modes separate, because $\mathbf{k}_{\mathrm{sp}}(0, \pm 1)$ lies in the $(x, y)$ plane whose magnitude differs from that of the $\mathbf{k}_{\mathrm{sp}}(-1,0)$ and $\mathbf{k}_{\mathrm{sp}}$ $(1,0)$ modes which lie in the $x$ axis. The vector $\mathbf{k}_{x}$ increases with the incident angle of the light that moves in the positive $x$ direction; $\mathbf{k}_{\mathrm{sp}}(-1,0)$ in the negative $x$ direction become less negative, according to Eq. (1), and $\mathbf{k}_{\mathrm{sp}}(1,0)$ becomes more positive. These results suggest that the $(-1,0) \mathrm{Ag} / \mathrm{Si}$ mode shift to longer wavelength and lower energy, but the $(1,0) \mathrm{Ag} / \mathrm{Si}$ mode does the opposite. The vectors $\mathbf{k}_{\mathrm{sp}}(0, \pm 1)$ lie in the $(x, y)$ plane, and the $(0, \pm 1) \mathrm{Ag} / \mathrm{Si}$ mode moves to shorter wavelength and higher energy. At a small incident angle, Bragg scattering in the $x$ direction causes both forward and backward SPP waves to interfere with each other and set up two standing waves. Besides, strong scattering of the incident light makes the $\mathbf{k}_{\mathrm{sp}}(1,0)$ mode weaker than the $\mathbf{k}_{\mathrm{sp}}$ $(-1,0)$ mode. At an incident angle of $\theta$ from the $x$ direction, the electric field polarized in $y$ direction exceeds that polarized in the $x$ direction. $\mathbf{k}_{\mathrm{sp}}(0, \pm 1)$ was excited by an electric field polarized in the $y$ direction, so the transmission intensity of $\mathbf{k}_{\mathrm{sp}}(0, \pm 1)$ exceeded that of $\mathbf{k}_{\mathrm{sp}}(-1,0)$. Figure 4(b) shows the transmission spectra when the square holes are smaller than a half lattice constant $a / 2$, and the $(0, \pm 1)$ $\mathrm{Ag} / \mathrm{Si}$ and $(-1,0) \mathrm{Ag} / \mathrm{Si}$ modes split, such that the intensity of $(-1,0) \mathrm{Ag} / \mathrm{Si}$ exceeds that of $(0, \pm 1) \mathrm{Ag} / \mathrm{Si}$ at $\theta<10^{\circ}$. When $\theta>12^{\circ}$, the intensity of the $(0, \pm 1) \mathrm{Ag} / \mathrm{Si}$ mode exceeds that of the $(-1,0) \mathrm{Ag} / \mathrm{Si}$ and $(1,0) \mathrm{Ag} / \mathrm{Si}$ modes, because incident light at a small incident angle underwent strong scattering, resulting in the very high transmission intensity of backward SPP waves. However, transmission intensity of forward SPP waves increases with larger projection quantity in the direction of propagation direction. The transmission intensity of the backward $\mathbf{k}_{\mathrm{sp}}(-1,0)$ mode is simultaneously lower than that of the $\mathbf{k}_{\mathrm{sp}}(0, \pm 1)$ mode. The $(1,0) \mathrm{Ag} / \mathrm{Si}$ mode is observed at a smaller angle in Fig. 4(b) than that associated with the larger hole size in Fig. 4(a). A surface charge field of a larger hole in the $\mathbf{k}_{\mathrm{sp}}(1,0)$ mode forms less easily than a smaller square hole, so a larger $\mathbf{k}_{x}$, and thus $\theta$, is required to the support $\mathbf{k}_{\mathrm{sp}}(1,0)$ mode. For some range of $\theta$, the $\mathbf{k}_{\mathrm{sp}}(-1,0)$ mode forms more easily than the $\mathbf{k}_{\mathrm{sp}}(0, \pm 1)$ mode, indicating that the surface charge field changes between the $(0, \pm 1) \mathrm{Ag} / \mathrm{Si}$ mode and the $(-1,0)$ $\mathrm{Ag} / \mathrm{Si}$ mode.

In conclusion, when normal incident light propagates through the sample, the Bragg scattering of SPP on periodic perforated hole arrays generates two standing waves $\left(\omega_{+}\right.$and $\left.\omega_{-}\right)$and the photonic band gap. The SPP dispersion relations with variously sized square holes were measured to investigate the surface charge field at the periodic metal array. The intensity of the $(0, \pm 1) \mathrm{Ag} / \mathrm{Si}$ mode exceeds that of the $(-1,0) \mathrm{Ag} / \mathrm{Si}$ when the hole size is sufficiently large. The surface charge density of the $(0, \pm 1) \mathrm{Ag} / \mathrm{Si}$ mode exceeds that of the $(-1,0) \mathrm{Ag} / \mathrm{Si}$ mode with a smaller metal line width, explaining why transmission intensity is higher.

This work is supported by the National Science Council of Republic of China under Contact No. NSC 94-2215-E002-042.

${ }^{1}$ H. F. Ghaemi, Tineke Thio, D. E. Grupp, T. W. Ebbesen, and H. J. Lezec, Phys. Rev. B 58, 6779 (1998).

${ }^{2}$ T. W. Ebbesen, H. J. Lezec, H. F. Ghaemi, T. Thio, and P. A. Wolff, Nature (London) 391, 667 (1998).

${ }^{3}$ H. J. Lezec, A. Degiron, E. Devaux, R. A. Linke, L. Martin-Moreno, F. J. Garcia-Vidal, and T. W. Ebbesen, Science 297, 820 (2002).

${ }^{4}$ Liang-Bin Yu, Ding-Zheng Lin, Yi-Chun Chen, You-Chia Chang, Kuo-Tung Huang, Jiunn-Woei Liaw, Jyi-Tyan Yeh, Jonq-Min Liu, Chau-Shioung Yeh, and Chih-Kung Lee, Phys. Rev. B 71, 041405(R) (2005).

${ }^{5}$ W. L. Barnes, T. W. Preist, S. C. Kitson, and J. R. Sambles, Phys. Rev. B 54, 6227 (1996).

${ }^{6}$ S. C. Kitson, W. L. Barnes, and J. R. Sambles, Phys. Rev. B 52, 11441 (1995).

${ }^{7}$ D. Egorov, B. S. Dennis, G. Blumberg, and M. I. Haftel, Phys. Rev. B 70, 033404 (2004).

${ }^{8}$ Shaum M. Williams, Amanda D. Stafford, Kenneth R. Rodriguez, Trisha M. Rogers, and James V. Coe, J. Phys. Chem. B 107, 11871 (2003).

${ }^{9}$ Shaun M. Williams, Amanda D. Stafford, Trisha M. Rogers, Sarah R. Bishop, and James V. Coe, Appl. Phys. Lett. 85, 1472 (2004).

${ }^{10}$ Y.-H. Ye and Jia-Yu Zhang, Appl. Phys. Lett. 84, 2977 (2004).

${ }^{11}$ M. A. Ordal, L. L. Long, R. J. Bell, S. E. Bell, R. R. Bell, R. W. Alexander, Jr., and C. A. Ward, Appl. Opt. 22, 1099 (1983). 